\title{
Medical, Economic Issues in Combating the Pandemic Caused by Sars-Cov-2 Coronavirus in the Russian Federation
}

\author{
G. R. Bayramgulova*, A. B. Zulkarnaev, S. M. Muzafarov, G. A. Yagafarova, \\ F.G. Aminev
}

Federal State Budgetary Educational Institution of Higher Education Bashkir State University, Sibay, Russia ${ }^{*}$ Corresponding author. Email: gulfira-medina@mail.ru

\begin{abstract}
According to history, pandemics and epidemics accompanied the humanity. The human community overcame the epidemics of typhoid, cholera, smallpox, plague, poliomyelitis. The listed diseases have gone down in history as infections affected by mankind. These successful victories were achieved thanks to successful development of medicine, epidemiology, and vaccination. In the organization of Russian health care, the coronavirus infection pandemic required additional changes, economic reforms, and amendments to the health care system. Epidemiologists believe that only widespread vaccination and the conscientious implementation of preventive measures by every resident can stop the COVID-19 pandemic.
\end{abstract}

Keywords: pandemic, coronavirus infection COVID-19, vaccines, epidemiology

\section{INTRODUCTION}

Pandemics have brought chaos around the world throughout human history, changing the course of events in the world order. Diseases such as malaria, plague, smallpox, SARS, and Ebola have claimed the lives of millions and become part of history. The famous plague of Galen was a long-standing pandemic that affected Italy, Egypt, Asia Minor, Greece. This unknown disease was brought to Rome by ordinary soldiers returning from Mesopotamia around 165 AD. Unbeknownst to the soldiers, they spread a contagious disease, which subsequently devastated the Roman army, killing about 5 million people, which made up a third of the population. The pandemic has affected all areas of social life of the human community. Spread of the coronavirus infection COVID-19 has spread to the countries of the world community. First of all, the COVID-19 coronavirus infection affected the healthcare system, namely, from the position of its potential to effectively respond to the situation with the spread of viral infection $[1,2,3,6,7]$.

\section{MATERIALS AND METHODS}

The subject of our study was an overview of the main anti-epidemic and economic issues in the fight against coronavirus infection. To find answers to medical and economic questions, we studied the principles of arranging the Russian health care in the context of a coronavirus infection pandemic, as well as medical aspects of the fight against the SARS-CoV-2 coronavirus.

\section{RESULTS AND DISCUSSION}

In the organization of Russian health care, the coronavirus infection pandemic required additional changes, economic reforms, and amendments to the health care system. The shortage of bed space in hospitals for infectious patients, equipment for mechanical ventilation, a shortage of doctors, nurses and diagnostic equipment have become the priority issues for solving in the healthcare system.

The problem also affected the organization of pharmacy, the base of which, as it turned out, did not fully meet the needs of the country in emergency situations. In a short period of time, some of the drugs 
for treatment of acute respiratory infections disappeared from the assortment of the pharmacy chain, and later they appeared, but at an inflated cost.

Academician of RAS V.I. Starodubov with colleagues in his analytical report pointed out the main factors affecting the financial situation of medical institutions in the context of the spread of coronavirus infection: presence of accounts payable of medical institutions over the past periods, the impact on the economy and health care of the fall in oil prices, problems associated with the collection budgetary funds and funds in the compulsory health insurance system, gaps in the income of medical organizations for compulsory medical insurance and for paid services, an increase in prices for goods and services provided by medical institutions $[4,5]$.

COVID-19 is an infectious disease caused by the SARS-CoV-2 coronavirus. The incubation period varies from 2 days to two weeks; on it is average 5-7 days. The period of illness is 9-11 days. The rehabilitation process is from 2 weeks to 1 month. Rehabilitation is individual and in some cases may take longer.

The incubation period of COVID-19 is up to two weeks, but most often the disease manifests itself 5-7 days after contact with an infected person. The main symptoms of COVID-19:

- The earliest symptomatic symptom of COVID19 is isolated loss of smell without nasal congestion. In $80 \%$ of cases, there is also a loss of taste.

- $\quad$ Subfebrile temperature up to $37.0-37.5^{\circ} \mathrm{C}$, mild cough, headaches, sputum discharge, weakness, fatigue, drowsiness, muscle pain, aches, shortness of breath;

- In covid-positive patients, the respiratory rate is more often than 22 times per minute. As a rule, the most noticeable feeling of lack of air develops by 6-8 days from the moment of illness;

- Decreased oxygen saturation or blood oxygen saturation (normally, in young people without concomitant pathologies, it shall be at least $95 \%$ ). This indicator can and shall be monitored independently with a pulse oximeter;

- Diarrhea, blurred vision and conjunctivitis, increased skin sensitivity to touch.

For an asymptomatic course of infection, it is characteristic that $80 \%$ of those infected with confirmed analyzes of studies are not included in the official statistics of the spread of infection. Therefore, absence of symptoms does not guarantee absence of health problems, and in such cases, subclinical changes in the lungs can occur, which can be observed on computed tomography (CT). There are asymptomatic carriers, and their number may be at least $40-45 \%$ of all infected. In mid-April of 2020, about $30 \%$ of those infected in Russia and $52 \%$ in Moscow transferred SARS-CoV-2 without obvious symptoms. In Italy, where almost the entire population was examined, asymptomatic carriers of the coronavirus turned out to be $42.5 \%$ of the total number of those infected. In addition, as practice shows, immunity in asymptomatic carriers of the virus lasts less and works weaker than in those who have been ill.

A PCR test or a PCR smear (polymerase chain reaction test) is one of the main diagnostic tools for COVID-19. This study is most informative from 1 to 5 days of the disease, but in $30-40 \%$ of cases it gives a false-negative result. It is taken for examination by a polyclinic doctor. It is important to know that the smear shall be taken with two different sticks: one - from the nose until the characteristic sign "to tears" appears, the second - from the back of the throat, without touching the tongue and the inner surface of the cheeks.

Blood tests in patients with COVID-19 show the following changes: lymphocytopenia in $80 \%$ of patients; thrombocytopenia in $35 \%$ of patients; leukopenia in $30 \%$ of patients; ESR indicators are increased; ferritin is increased; C-reactive protein is elevated. C-reactive protein is a major marker of pulmonary activity with COVID. Its increase reliably shows the volume of damage to the lung tissue. The higher the C-reactive protein in COVID, the greater the lesion. An experienced physician can tell the percentage of lung damage based on C-reactive protein levels. Low oxygen levels in the blood indicate lung damage. Saturation indicators can guide a doctor in identifying pneumonia in the early stages of coronavirus infection. Measurements of oxygen saturation with a pulse oximeter are carried out at rest, as exercise can lower the oxygen saturation of the blood. If females having a manicure with nail polish, the saturation index may be mistakenly reduced by $5-10 \%$.

Computed tomography of the chest organs is the most reliable diagnostic method. Detection of bilateral pneumonia with a percentage of damage up to $25 \%$ will not affect further actions in any way, and such a affect occurs in $80 \%$ of cases. CT is not done even at the first signs of COVID-19, since if there is a affect, it will be detected on the 5-7th day. Repeated CT scan, more precisely CT angiography of the lungs, is done only in case of a sharp deterioration in the condition. The aim of the study is to identify possible pulmonary embolism.

In tests for antibodies in human blood, 2 types of antibodies to COVID-19 can be produced: IgM show that the patient has recently had a disease, appear 7 days after the onset of the first symptoms and can last 23 weeks, even when the patient recovers, these antibodies disappear in within 2-3 months; IgG appears 3 weeks after the illness and can be present in the blood for 2 to 4 months, protecting the body from re-infection. 
There is evidence that antibodies can last longer. Antibody levels depend on the severity of COVID-19. In patients with a mild form of the infection, the antibody content is lower than in those with a severe form. Most of those who have been ill in a mild form have a low level of antibodies to the virus and the $\mathrm{IgG}$ indicator decreases rather quickly. Persistent immunity is formed when the IgG antibody index is more than 8.5. This amount of antibodies is sufficient to prevent reinfection.

There are two dangerous coronavirus storms: bradykinin and cytokine.

- A cytokine storm consists in the fact that a large number of inflammatory mediators are produced in the patient's body. This is the reason for the increase in temperature, which is not stopped by antipyretic drugs, can cause damage to all organs. A similar condition can develop on the 5-7th day of the disease. In clinical practice, it is stopped by hormonal antiinflammatory drugs, which have shown the greatest efficiency in severe disease. Such treatment is used under the supervision of a physician, as it has many contraindications, including diabetes. Also, in a hospital setting, a number of other drugs from the group of cytokine storm blockers are used [8].

- A bradykinin storm is an increase in concentration of bradykinin, which dilates blood vessels and, therefore, lowers blood pressure and increases vascular permeability. Increase in the concentration of bradykinin and contribute to many of the symptoms of patients with COVID-19, for example, appearance of a dry cough, fatigue. As a consequence, tissue fluid with hyaluronic acid form a hydrogel in the lumen of the alveoli of the lungs and cause respiratory failure, causing the ineffectiveness of artificial ventilation (ALV). On a computed tomography scan of the chest, this represents areas of the lungs with a "ground glass" effect. In $95 \%$ of cases, this affect is not bacterial pneumonia and is not treated with antibiotics. Antibacterial drugs are used in cases of complications of pneumonia with a bacterial infection. That is why infusion therapy is not carried out without special indications, as it causes additional pulmonary and cerebral edema.

Covid-19 in a mild form is treated by analogy with an acute respiratory viral infection: adherence to the drinking regimen, at least 2 liters of warm drink per day; use of antiviral drugs as directed by a doctor; when the temperature rises above $38.5^{\circ} \mathrm{C}$, it is worth starting to take antipyretic drugs. If the paracetamol does not reduce a fever, then a cytokine storm has probably begun, in this case, vitamin $\mathrm{D}$ is prescribed in a prophylactic dosage of $4000 \mathrm{IU}$, which supports the regulation of the immune response in order to avoid an overreaction that occurs in patients with a severe course of coronavirus infection. A study by American scientists showed that people with vitamin D deficiency have an almost 2 times higher risk of testing positive for COVID-19. At the moment, there is no drug that would selectively affect the causative agent of the viral infection COVID-19. If a patient has concomitant diseases, then with a coronavirus infection, they are exacerbated. Therefore, the treatment is symptomatic. Antibiotic therapy is prescribed when a bacterial infection is connected, which occurs in $5 \%$ of cases, their appointment shall be based on laboratory blood tests -- a manifestation of leukocytosis. The most prescribed antibiotic is Azithromycin. The only case when, in presence of viral pneumonia with lung damage, more than $20 \%$, antibiotics are prescribed for prophylactic purposes, is diabetes mellitus.

Favipiravir (Coronavir) has shown rapid clearance of the COVID-19 virus and faster clinical improvement. This drug has many contraindications and side effects.

There are a considerable number of factors influencing the spread of coronavirus, and, unfortunately, it is impossible to take everything into account. Mathematical models that calculate the spread of the virus are just predictions, which often turn out to be far from reality. The following factors can affect the spread of coronavirus: the cold season, other types of coronaviruses, as well as the causative agent of influenza and other infectious agents that are dependent on seasonal fluctuations and are more active during the cold season. Dry winter air promotes efficient transmission of viruses. Moreover, in winter, people are more often in confined spaces, where the coronavirus is transmitted more efficiently than in the open air. Influenza infection can worsen the complications of COVID-19. A seasonal outbreak of influenza occurs in late autumn, in winter, which can complicate the epidemiological situation with the coronavirus. Conversely, COVID-19 can negatively affect the spread of influenza. With increased attention to the coronavirus pandemic, it may be more difficult to prevent a seasonal flu outbreak.

The first vaccination in Russian history against a serious smallpox disease was given to Catherine II back in 1768. In the 10th century, the smallpox epidemic reduced the population of Europe and Russia, and thanks to the vaccination, smallpox was successfully affected and went down in the history of epidemiology. At the moment, a solid number of different vaccines against COVID-19 are in development. A record 65 days passed between the sequencing (determination of elementary particles) of SARS-CoV-2 and the first injection of a test vaccine into a volunteer. In the history of mankind, a vaccine has not yet been developed in a short time. In a pandemic, as in an emergency, such a rapid ramp-up in vaccine development is imperative. Russian vaccine recommended for use Gam-COVIDVac, trade mark Sputnik V. This is the world's first 
vaccine against coronavirus offered by the National Center for Epidemiology and Microbiology of Russia. It is entered twice, with an interval of three weeks. A Russian vaccine recommended for use by EpiVacCorona, which in the body promotes the formation of protective immunity against the SARSCoV-2 coronavirus as a result of two intramuscular administrations with an interval of 14-21 days. At the research stage, the vaccine of the Research Institute of Vaccines and Sera FMBA of Russia (Federal Medical and Biological Agency). 2time immunization within 14 days is considered. At the stage of the beginning the application of the vaccine of the Federal Scientific Center for Research and Development of Immunobiological Preparations named after M.P. Chumakov, RAS, Covivac, is used in two administrations with an interval of two weeks.

Epidemiologists believe that only widespread vaccination and the conscientious implementation of preventive measures by every resident can stop the COVID-19 pandemic.

In 2021, free voluntary vaccination started in Russia. The psychological attitude towards vaccination is no less important, because extreme excitement can provoke an increase in blood pressure. Due to the fact that there is still no exact data on the impact of COVID-19 disease on the effectiveness of the formation of immunity, Rospotrebnadzor recommends self-isolation before and after vaccination. As practice has shown, vaccinated persons tolerate the disease more easily. Vaccination promotes the synthesis of specific antibodies in the blood to the pathogen, thereby preparing a person to face a coronavirus infection.

\section{CONCLUSION}

Therefore, according to history, pandemics and epidemics have accompanied humanity. The human community overcame the epidemics of typhoid, cholera, smallpox, plague, poliomyelitis. The listed diseases have gone down in history as infections affected by mankind. These successful victories were achieved thanks to successful development of medicine, epidemiology, and vaccination. In the organization of Russian health care, the coronavirus infection pandemic required additional changes, economic reforms, and amendments to the health care system. Epidemiologists believe that only widespread vaccination and the conscientious implementation of preventive measures by every resident can stop the COVID-19 pandemic.

\section{REFERENCES}

[1] G. R. Bayramgulova, G. U. Ishmullina, A.G. Aitkulova. An Assessment of the coronavirus disease in the world at the beginning of 2020 , International scientific-practical conference
"Theoretical and Applied Problems of Modern Science and Education", Kursk, 2021.

[2] V. I. Pokrovsky, O. I. Kiselev, P. G. Nazarov. SARS: severe acute respiratory syndrome. New virus, new disease. Cytokines and Infl ammation. 2(2) (2003) pp. 42-51.

[3] B. K. Romanov. Coronavirus infection COVID2019. Safety and risk of pharmacotherapy. 8(1) (2020) pp. 3-8. DOI: https://doi.org/10.30895/2312-7821-2020-8-1-3-8

[4] V. I. Starodubov, F. N. Kadyrov and etc. Analytical report: "The Impact of the Covid-19 Coronavirus on the situation in Russian healthcare", Moscow, 2020. https://mednet.ru/

[5] V. M. Timerbulatov, M. V. Timerbulatov. Health care during and after the Covid-19 Pandemic, Bulletin of the Academy of Sciences of the Republic of Bashkortostan, 2 (2020) p. 77.

[6] C. I. Paules, H. D. Marston, A. S. Fauci. Coronavirus infections - more than just the common cold. JAMA. 323(8) (2020) pp.707-8. DOI: https://doi.org/10.1001/jama.2020.0757.

[7] J. Liu, X. Zheng, Q. Tong, W. Li, B. Wang, Sutter $\mathrm{K}$, et al. Overlapping and discrete aspects of the pathology and pathogenesis of the emerging human pathogenic coronaviruses SARS-CoV, MERSCoV, and 2019-nCoV. J Med Virol. 2020. DOI: https://doi.org/10.1002/jmv.25709.

[8] L. J. Stockman, R. Bellamy, P. Garner. SARS: systematic review of treatment eff ects. PLoS Med. 3(9) (2006) e343. DOI: https://doi. org/10.1371/journal.pmed.0030343.

[9] The largest deadly pandemics and epidemics in human history, https://www.infoniac.ru/news/Samyesmertonosnye-pandemii-i-epidemii-v-istoriichelovechestva.html

[10] Vaccination history in Russia, http://cgon.rospotrebnadzor.ru/content/33/3706 